\title{
Review of: "Control of particulate nitrate air pollution in China"
}

Bin Zhu

Potential competing interests: The author(s) declared that no potential competing interests exist.

The paper simplified, the complex physical and chemical processes related to lifetime and concentration of particle nitrate ([NO3-]-particle) into 3 equations. From the equations we can easily comprehend the sensitivity of [NO3-]-particle to the changes of the ratio of [NO3-]- particle/ [NO3-]- (gas+particle) and their depositions. I think only these formulae could explain that the lack of response of nitrate to NOx emission controls in winter and even increased during winter haze pollution events in the North China Plain, also verified by chemical transport model. 